\title{
Cardiac Resynchronization Therapy in Patients with Atrial Fibrillation
}

\author{
Mohammad Ramadan ${ }^{1}$ and Marwan Refaat ${ }^{1}$ \\ ${ }^{1}$ American University of Beirut Medical Center
}

July 7, 2020

Atrial fibrillation (AF) is the most common cardiac arrhythmia and often occurs with heart failure (HF) [1]. AF prevalence increases with increasing severity of HF: for instance its prevalence ranges from 5 percent in patients with New York Heart Association (NYHA) functional class I HF to 40 percent in patients with NYHA class IV HF [2]. Its presence with HF plays a significant prognostic role and increases morbidity and mortality. Heart Failure with reduced ejection fraction (HFrEF) is associated with cardiac arrhythmias [3]. $\mathrm{HFrEF}$ is also one of the indications for Cardiac resynchronization therapy (CRT) placement [4]. Therefore, many patients undergoing CRT implantation will concomitantly have HF and AF. As the benefit from CRT in HF patients has been established, the data on patients with both HF and AF is limited, because patients with atrial arrhythmias were excluded from most of the major CRT trials, such as CARE-HF and COMPANION [5]. However, a number of observational studies and small randomized clinical trials suggest a benefit from CRT in AF and HF patients such as a CRT-mediated ejection fraction (EF) increase [6, 7]. Other studies showed a high non-response rate in patients with AF as compared to those in sinus rhythm (SR) [8]. Thus, it is important to determine whether CRT has a beneficial role in these patients to decide on adding an atrial lead at the time of CRT implantation especially in patients with longstanding-persistent $\mathrm{AF}$.

In their published study, Ziegelhoeffer et al. investigated the outcomes of CRT placement with an atrial lead in patients with HF and AF. This was done by conducting a retrospective analysis of all patients with AF who received CRT for HF at the Kerckhoff Heart Center since June 2004 and were observed until July 2018- completing a 5-year follow-up. The authors identified 328 patients and divided them into 3 subgroups: paroxysmal (px) AF, persistent (ps) AF, and longstanding-persistent (lp) AF, with all patients receiving the same standard operative management. During the observation period, the authors analyzed the rhythm course of the patients, cardiac parameters (NYHA class, MR, LVEF, left atrial diameter) and performed a subgroup analysis for patients who received an atrial lead. The study showed that all groups had a high rate of sinus rate (SR) conversion and rhythm maintenance at 1 and 5 years. Specifically, the patients who received an atrial lead among the lp AF group were shown to have a stable EF, less pronounced left ventricular end-systolic diameter (LVESD) and left ventricular end diastolic diameter (LVEDD) and lower mitral regurgitation (MR) rates at one year follow-up as compared to the group without atrial lead placement. Moreover, the results of the lp group were similar to the ps-AF group, although the latter had a lower number of participants $(n=4)$ without initial implantation of the atrial lead. The authors attributed the improvement in cardiac function and SR conversion to CRT and the implantation of an additional atrial lead.

Although some studies showed that CRT therapy reduced secondary MR in HF [9, 10], this study additionally suggests that CRT with an atrial lead was associated with improved myocardial function and improvement of interventricular conduction delay triggering cardiac remodeling in patients with $\mathrm{HF}$ and $\mathrm{AF}$. Although the results showed better cardiac function in the subgroup analysis of the patients with an additional atrial lead, these results were reported as percentages with no level of significance specified, hence statistical significance 
of the difference in the described parameters (such as LVESD, LVEDD) could not be determined. Further investigation via prospective studies is needed with larger sample size in the future to further support the results of the study especially that it was done in a single center and had a relatively small sample size.

References:

1. Chung MK, Refaat M, Shen WK, et al. Atrial Fibrillation: JACC Council Perspectives. J Am Coll Cardiol. Apr 2020; 75 (14): 1689-1713.

2. Maisel, W.H. and L.W. Stevenson, Atrial fibrillation in heart failure: epidemiology, pathophysiology, and rationale for therapy. Am J Cardiol, 2003. 91 (6a): p. 2d-8d.

3. AlJaroudi WA, Refaat MM, Habib RH, et al. Effect of Angiotensin Converting Enzyme Inhibitors and Receptor Blockers on Appropriate Implantable Cardiac Defibrillator Shock: Insights from the GRADE Multicenter Registry. Am J Cardiol Apr 2015; 115 (7): 115(7):924-31.

4. Yancy, C.W., et al., 2013 ACCF/AHA guideline for the management of heart failure: a report of the American College of Cardiology Foundation/American Heart Association Task Force on Practice Guidelines. J Am Coll Cardiol, 2013. 62 (16): p. e147-239.

5. Cleland, J.G., et al., The effect of cardiac resynchronization on morbidity and mortality in heart failure. N Engl J Med, 2005.352 (15): p. 1539-49.

6. Leclercq, C., et al., Comparative effects of permanent biventricular and right-univentricular pacing in heart failure patients with chronic atrial fibrillation. Eur Heart J, 2002. 23 (22): p. 1780-7.

7. Upadhyay, G.A., et al., Cardiac resynchronization in patients with atrial fibrillation: a meta-analysis of prospective cohort studies. J Am Coll Cardiol, 2008. 52 (15): p. 1239-46.

8. Wilton, S.B., et al., Outcomes of cardiac resynchronization therapy in patients with versus those without atrial fibrillation: a systematic review and meta-analysis. Heart Rhythm, 2011. 8 (7): p. 1088-94.

9. van Bommel, R.J., et al., Cardiac resynchronization therapy as a therapeutic option in patients with moderate-severe functional mitral regurgitation and high operative risk. Circulation, 2011.124 (8): p. 912-9.

10. Breithardt, O.A., et al., Acute effects of cardiac resynchronization therapy on functional mitral regurgitation in advanced systolic heart failure. J Am Coll Cardiol, 2003. 41 (5): p. 765-70. 